NASA Technical Memorandum 83630

\title{
Measurement of Fluid Properties Using Rapid-Double-Exposure and Time-Average Holographic Interferometry
}

Arthur J. Decker

Lewis Research Center

Cleveland, Ohio

Prepared for the

Twentieth Joint Propulsion Conference

cosponsored by the AIAA, SAE and ASME

Cincinnati, Ohio, June 11-13, 1984 
MEASUREMENT OF FLUID PROPERTIES USING RAPID-DOUBLE-EXPOSURE AND TIME-AVERAGE HOLOGRAPHIC INTERFEROMETRY

\author{
Arthur J. Decker \\ National Aeronautics and Space Administration \\ Lew1s Research Center \\ Cleveland, Oh10 44135
}

\section{Abstract}

The objective of this paper is to discuss the holographic recording of the time history of a flow feature in three dimensions. The paper begins by describing the use of diffuse illumination holographic interferometry for the three dimensional visualization of flow features such as shock waves and turbulent eddies. The double-exposure and $t$ Ime-average methods are compared using the characteristic function and the results from a flow simulator. A time history requires a large hologram recording rate. Results of holographic cinematography of the shock waves in a flutter cascade are presented as an example. Future directions of this effort, including the avallability and development of suitable lasers, are discussed.

\section{Nomenclature}

$I, I_{\max }, I_{\min }$
$\vec{k}$
$\vec{r}$
$T$
$V$
$\Delta \phi(\vec{k}, \vec{r}, t)$
$\Delta \phi(\vec{k}, \vec{r})$
$\Delta \phi_{t}(t)$
$\theta x, \theta y$

intensity

ray direction

position

exposure time

interference-fringe visibility

variation of phase of ray (eq. (3))

amplitude of phase variation (eq.

time variation of phase (eq. (5))

directional parameters (eqs. (6)

and (7))

$\mu(\vec{r}, \vec{k})$

characteristic function (eq. (3))

p

fluid density

\section{Introduction}

For more than 15 years, diffuse-1llumination holographic interferometry has been used for measurements in the propulsion field. (1) One appil$c$ ation has been the measurement and visualization of fluid properties. A very useful effect of diffuse illumination interferometry is interference fringe localization. When diffuse-illumination interferograms are recorded of certain flow features such as shock waves in compressors and cascades, the interference fringe pattern is observed to be located three dimen $\$ 2$ nally nearby, on, or
within the flow feature. $(2-4)$ The flow feature is made visible by this process.

Typically the interference fringe pattern is caused by the time variation of the density field of the fluid. But time variations caused by spectral changes, doppler shifts, and the like will also affect the fringe pattern. The processes of fringe formation and localization haye very general properties, sumarized best by vest. (5)
One very useful property occurs when the variations of the density $\rho(\vec{r}, t)$ with position $\vec{r}$ and with time $t$ are separable, that is when

$$
\Delta o(\vec{r}, t)=o_{r}(\vec{r}) o_{t}(t)
$$

Then the localization property is not expected to depend upon the particular method of holographic interferometry. That is, fringe localization should be the same for real time, double exposure, or time average holography, and for time average holography with modulation of the reference and object waves. (6) All of these methods are being used and investigated at Lewis Research Center, and are discussed in this paper.

In fact, given enough motivation, one can calculate the fringe formation and localization properties of any flow feature (or combination of flow features). That motivation has been missing in part because of inadequate hardware (particularly lasers) for holographic interferometry in a propulsion environment. Now, there are laser systems avallable that should make another hard look at diffuse illumination interferometry worthwhile.

Specifically, if enough holograms can be recorded per second, then the time history of an appropriate flow feature $c$ an be viewed three dimensionally. It has been demonstrated at Lewis that a 20 pulse-pair-per-second, double pulse, Q-Switched Nd:YAG laser operated at its second harmonic frequency of $532 \mathrm{~nm}$ can be used for holographic cinematography of time varying processes. (7) The use of this technique for the stroboscopic visualization of the lambda shock waves in a flutter cascade is described herein. Subsequently, an improved Nd:YAG laser for this application has become an off-the-shelf item.

The flashlamp-pumped dye laser can be operated at about the same pulse repetition rate as the Nd YAG laser. In addition, however, it can radiate large amounts of energy in long pulses. The pulse lengths are correct for time-average holography of the flows encountered in aircraft propulsion systems. This application of the flashlamp-pumped dye laser, which is being investigated at Lewis Research Center, is discussed briefly hẹrein.

\section{Fringe Formation and Localization}

Recording and Viewing an Interference Fringe

The application of diffuse-illumination, holographic interferometry can be understood if a few facts and concepts are accepted. Detalled proofs and discussions are found in the references.

Holography is to be regarded as a technique for recording light waves existing at different $t$ imes for playback at the same time. Once the hologram is recorded, the concept of holography is irrelevant to any interpretation or analysis. The 
holographic recording process as we use it is discussed extensively in the literature. $(8,9)$

The interference of the multiple light waves is considered in the geometrical optics approximation. The limitations of this approximation in interfergmetry have been discussed by Howes and Buchele. (10) In this approximation, it is light rays that are reconstructed in the holographic process. The rays are considered to be perpendicular to wave fronts. For a time varying fluid, the phase of the light wave changes along each ray during the exposure or multiple exposure recorded holographically. We consider only the case where the laser illumination of the fluid has passed through a diffuser. It is the interference of the multiple exposures, one light ray at a time, that is the foundation of diffuse illumination interferometry. In fact, the cross interferences between different light rays is a random process which does not yield high visibility interference fringes. The local visibility $V$ of an interference fringe pattern is defined as

$$
V=\frac{I_{\max }-I_{\min }}{I_{\max }+I_{\min }}
$$

where $I_{\max }$ and $I_{\min }$ are the local maximum and minimum intensitics in the pattern. Cross interferences between different rays are ignored.

The interference patterns of the different rays are viewed three dimensionally by rmaging points in the reconstructed image with a lens or other imaging system (see Fig. 1).

Each ray in diffuse-illumination holographic interferometry contributes its own intensity. That $1 \mathrm{~s}$, the contributions are added incoherently. The rays from a particular direction form an interference pattern. Rays from different directions in general form different patterns. A high visibility composite pattern is formed only when the individual patterns register in the image plane. That is one statement of the condition for fringe localization.

The viewing properties of a well localized interference fringe pattern are much like those of a three-dimensional opaque object. This fact is emphasized in Fig. 1 by showing a pattern that appears to a viewer to be in the virtual image space of a hologram of a fluid. When the pattern is caused by a flow feature and is located nearby, on, or within the flow feature, the pattern provides three-dimensional visualization of that feature.

The conditions for fringe formation and localization can be stated more quantitatively.

\section{Localization Condition}

Characteristic Function. Consider a light ray whose direction is given by the unit vector $\vec{k}$. Let the light ray pass through a point $\vec{r}$ in a flurd. Let the imaging system of $F_{1 g} .1$ be focused on that point. If a hologram is recorded of that light ray during a time interval $T$, then the phase factor of the reconstructed wave along that ray direction is proportional to

$$
\mu(\vec{r}, \vec{k})=\frac{1}{T} \int_{0}^{T} \exp \{j[\Delta \phi(\vec{k}, \vec{r}, t)]\} d t
$$

where $\Delta \phi(\vec{k}, \vec{r}, t)$ is the phase change along the ray having the direction $\vec{k}$ and passing through point $\vec{r}$, and

$$
\Delta \phi(\vec{k}, \vec{r}, 0)=0
$$

For rays confined to direction $\vec{k}$, and for points on some surface, the intensity in the observed interference pattern is proportional to

$$
I=|\mu(\vec{r}, \vec{k})|^{2}
$$

The function $\mu(\vec{r}, \vec{k})$ is called the characteristic function, and can be used to define all the methods of diffuse-illumination holographic interferometry.

Separable Flow Feature. There are expansions of Eq. (3) which can be used to treat completely, general time and spatial varıations of phase. (11) The following is restricted to flows which are separable or at least locally separable. Then, by definition, the change in phase can be written as

$$
\Delta \phi(\vec{k}, \vec{r}, t)=\Delta \phi(\vec{k}, \vec{r}) \Delta \phi_{t}(t)
$$

The change in phase along a ray is proportional to the integral along that ray of the change in fluid density. The change in phase will be separable if the change in flurd density is separable. Now, the characteristic function can be written expicitily as a function of $\Delta r(\vec{k}, \vec{r})$.

The condition for registration of the interference patterns in the plane of the imaging system of $F i g .1$ is that $|\mu[\Delta \phi(\vec{k}, \vec{r})]|$ not vary with direction $\vec{k}$. For an unrestricted viewing direction, this condition (to first order) reduces to two equations.

$$
\begin{aligned}
& \frac{\partial \Delta \phi}{\partial \theta}=0 \\
& \frac{\partial \Delta \phi}{\partial \theta_{y}}=0
\end{aligned}
$$

where $\theta_{x}$ and $\theta_{y}$ are angles in the planes containing the optical axis and the $x$ and $y$ axes. For convenience, the $x$ and $y$ axes can be in the plane of the diffuser, although other reference systems are possible. (3) The angles increase from the optical axis of the imaging system. In fact, any directional parameters may be used to evaluate the directional variations.

Together, Eqs. (6) and (7) define a spatial curve. Hence, in general, one expects to see a high visibility interference fringe pattern only in the neighborhood of a curve. The conditions actually observed may be less restrictive than this. If only one of EqS. (6) and (7) applies, then localization is on a surface. In any case, fringes are always observed over a finte volume of space, because of the nonzero depth-of-field of the imaging process. (5) 
Localization calculations must be done case-by-case.

\section{Shock-Wave Fringe Localization}

Examples of such calculations for shock wave surfaces have been reported in detail. These calculations were compared with data for the passage and bow waves of the blades of a rotating transonjc compressor stage at Lewis Research Center. $(3,4,12)$ The interference fringe position was compared with the shock wave position measured using laser anemometry. The calculation is algebraically complex even for the simple geometry of the shock wave, and the references should be read for detalls. some useful conclusions can be stated simply.

(1) The position of localization is a positional average weighted by the gradient of the change in fiuid density perpendicular to the axis of the imaging system. Fringes should localize near time varying features with large gradients of density (or refractive index).

(2) Still, even for shock waves, the interference fringe position may differ substantially from any position of the shock wave during the exposure. This difference is called fringe localization error.

(3) The fringe localization error is minimized by choosing a view which allows the shock wave to be viewed close to tangency.

(4) The fringe localization error is minimized by viewing shock wave surfaces which are curved.

(5) The fringe localization error is minimized by viewing shock waves with substantial positional variation in strength.

(6) The best image of the interference fringe will correspond to the minimum localization error. That is, the fringe visibility will be highest and the depth-of-field smallest for the minimum localization error.

Schumann has presented a result permitting conclusions similar to (2) through (6) to be drawn for any refractive index distribution. (13)

The key point is that given enough motivation, one can calculate the best localization and viewing properties for any flow feature. A model of the flow feature is required. For experimental work then, a range of viewing directions must be selected to handle possible deviations from the model.

Even for separable flows, the results will depend upon the holographic technique. The different holographic techniques are compared in the following section.

\section{Holographic Techniques}

\section{Sensitivity}

The sensitivity of time-average holography is about half that of the double exposure techniques. This conclusion is drawn by substituting the appropriate time functions into Eq. (5) and then by substituting Eq. (5) into Eq. (3).
Double-Exposure Holography. There are two versions of double-exposure hotography: truedouble- and rapid-double-exposure. For true-doubleexposure holography, the scene is time varying only between the two exposures. An example would be a steady flow versus no flow condition. In rapid-double-exposure holography, the flow varies steadily, but the exposures are so short that the density field can be considered constant during an exposure. An example is Q-switched laser holography of the passage shock waves of a compressor rotor. $(2-4)$ The exposure is measured in nanoseconds. For both cases, the characteristic function is the same:

$$
|u(\vec{r}, \vec{k})|^{2}=\cos ^{2}[\Delta \phi(\vec{r}, \vec{k}) / 2 .]
$$

where $\Delta \phi(\vec{r}, \vec{k})$ is the change in phase between the two exposures. Equation (8) is the familiar sinusoldal fringe pattern usually associated with interferometry. The same expression can be used for nonseparable flows, but then the change in phase should be labeled with the times of the two exposures.

Time-Average Holography. The characteristic function for time-average holography will depend upon the specific form of the time function $\Delta \phi t(t)$ in Eq. (5). The best known example is the sinusoidal motion of an object vibrating in a norma? mode. Then the characteristic function yields the familiar Bessel fringes. For flows, it is more realistic to choose, a phase that changes innearly with time. If $\Delta \phi(\vec{r}, \vec{k})$ is the total change in phase during the exposure, the interference fringe pattern is given by

$$
|u(\vec{r}, \vec{k})|^{2}=\sin ^{2} \frac{[\Delta \phi(\vec{r}, \vec{k}) / 2 \cdot]}{[\Delta \phi(\vec{r}, \vec{k}) / 2 \cdot]^{2}}
$$

This expression contains the familiar sinc function.

The key point is that the first zero or dark fringe in double-exposure holography occurs when

$$
\Delta \phi(\vec{r}, \vec{k})=\pi
$$

whereas, the first zero or dark fringe in timeaverage holography will not occur unt 11

$$
\Delta \phi(\vec{r}, \vec{k})=2 \pi,
$$

hence, the decrease in sensitivity.

Flow Simulator

The holographic techniques can be demonstrated with a flow simulator. The flow simulator is a $5 \times 5 \times 3 \mathrm{~cm}$ crystal of KD*P with pairs of 1-cm-wide electrodes attached to its $5 \times 5 \mathrm{~cm}$ top and bottom surfaces. Light can enter and exit its $5 \times 3 \mathrm{~cm}$ faces Its properties have been reported in detail.(14) Its principle application has been to test the frame-to-frame repeatability of a holographic motion picture setup. (7) The voltages on the four electrodes $c$ an be changed between exposures of a double-exposure hologram. The electrode-planes are perpendicular to the optic axis of the crystal. The refractive index distribution then changes between exposures for light 
polarized perpendicular to the optic axis. The same step in refractive index distribution from frame to frame can then be used to check repeatability of the holographic motion picture setup. The gradient of refractive index is primarily perpendicular to the optic axis, and the refractive index distribution is symetrical about the midplane of the crystal. Consequently, the interference fringes localize near the midplane of the crystal.

For evaluating a rapid-double-exposure setup with exposure separations ranging from 1 to 100 $\mu \mathrm{S}$, the refractive index $\mathrm{c}$ an be changed in nanoseconds. To evaluate a setup for tıme-average holography, the voltages on the electrodes are varied more slowly. The results reported immediately below were recorded in 16 to $31 \mathrm{~ms}$. An argon ion laser was used for lllumination. The time scale has no effect on the principles of holographic interferometry.

One problem with using the simulator for time average holography is that the phase change along a ray is typically $\pi$ or less, except near the electrodes. These changes yieid easily observed dark fringes in double exposure holography. But because of the reduced sensitivity discussed above, only the fringes near the electrodes appear in time-average holography. This problem can be overcome by using a double pass through the simulator. Another partial solution is to time modulate the reference (or object) wave during the exposure. The effect is to add another time varying function to the argument of the exponentral of Eq. (3) for the characteristic function. To first approximation, the result is equivalent to adding a constant phase change tothe change in distribution of phase $\Delta d(\vec{k}, \vec{r})$. The overall brightness of the hologram image is reduced. But the dark fringes are made visible. Because the positionally and directionally dependent part of the argument of Eq. (3) for the characteristic function does not change, the localization condition is not affected.

\section{Flow Simulator Results}

In Fig. 2, fringe patterns are shown for four different methods of diffuse illumination, holographic interferometry. The flow simulator discussed above was the subject. The change in phase distribution is the same for all methods. The potential differences on the left and right pairs of electrodes had opposite signs. Hence, the phase change ranges from positive on the left to negative on the right. The object illumination was derived from a collimated laser beam incident on a diffuser immediately in front of the simulator. The setup is the same as that described by Weimer.

Figure 2(a) shows a result for so called realtime, infinite-fringe holographic interferometry. A Newport Research thermoplastic holocamera was used to obtain the infinite-fringe condition. A hologram is recorded of the simulator before voltage is applied to the electrodes. The hologram image is reconstructed and the object 1 llumination turned on at the same time. The reconstructed object wave and the object wave interfere. With no voltage applied, the two waves are identical and no fringes appear. When voltage is applied, interference fringes characteristic of the change in phase distribution appear and are photographed directly. The fringes are localized near the midplane of the crystal as mentioned above.
Maintaining this infinite fringe condition (perfect alignment) would be difficult in a propulsion environment.

Figure $2(b)$ is from a double-exposure hologram of the same change shown in Fig. 2(a). The double exposure is recorded on a photographic plate. The fringe pattern is about the same as that of Fig. 2(a), but has better visibility. The only reason for the better visibility is that the direct object beam and the reconstructed object beam in Fig. 2(a) could not be adjusted for equal intensity, a requirement for maximum fringe visibility.

Figure $2(c)$ is from a time-average hologram where the phase changed gradually to that of Figs. $2(a)$ and (b). The change occurred gradually during the exposure $t$ ime of 31 ms. (The actual change was exponential rather than linear.) Because of the reduced sensitivity, fringes are observed only near the electrode positions. The fringes are similar to the fringes that would be observed at half the phase change in double-exposure holography.

Figure $2(d)$ is from a time-average hologram where the frequency of the reference beam was shifted by a moving mirror. During the exposure, the effect was equivalent to imposing an additional time-linear phase shift of $\pi$ on the phase distribution of the object. The fringe on the right side is cancelled, and the fringe on the left side is enhanced. Although the fringe is localized as before, its visibility is reduced.

In reference to $\mathrm{F} i \mathrm{~g} .2(\mathrm{~d})$, other means were also used successfully to overcome the reduced sensitivity of time-average holography. These means included phase modulation of the reference beam with an electrooptic phase modulator, a double pass, and time variation of the spectrum of the laser. The electrooptic modulator was a Pockels cell borrowed from the Q-Switch of a laser. The spectrum was made unstable by adjusting the posi$t$ ion of the argon laser etalon perpendicular to the laser axis. With the exception of the double pass through the simulator, the fringe visibility is reduced.

The double-pass method will be used to evaluate laser systems for time-average holographic cinematography. However, it is estimated that $a$ phase modulation amplitude up to $0.6 \pi$ can be used to selectively enhance the contrasts of flow features. Selectivity would be improved if the modulation were a function of $(\vec{k}, \vec{r})$ also. Possible modulators include electrooptic and acoustooptic modulators as well as moving mirrors and moving holographic optical elements.

The successful application of the principles outlined above depends upon the avallability of surtable laser systems. The objective at Lewis is to use holography for three-dimensional visualization and measurements of time varying fluids as they appear in propulsion applications. The requirement is for high-pulse-rate laser systems. For double-exposure holography, each pulse must actually consist of a pair of pulses. A typical separation of the pulses in a pair is 10 ws. For time-average holography, long pulses are used. The pulse length must be about twice that for the same application in double-exposure holography. 
Double-exposure holographic cinematography is discussed next.

\section{Rapid-Double-Exposure Holographic Cinematography in a Flutter Cascade}

Transonic Flutter Cascade

Rapid-double-exposure holography using a Q-switched ruby laser has been reported by Boldman, et al. for a flutter cascade. (15) The same cascade has been used to evaluate a holographic motion picture system. The linear cascade, as reported, consists of three two-dimensional airfoils driven to oscillate about the midchord axis in harmonic pitching motion. The frequency of oscillation is about $580 \mathrm{~Hz}$, the amplitude about $1.2^{\circ}$, and the mean angle of attack $6.0^{\circ}$. The freestream Mach number is 0.81 . Lambda-type shock waves appear near the leading edges of the airfolls. The continuously changing flow field is a convenient subject for rapid-double-exposure holography, as opposed to true-double-exposure holog$r$ aphy. The shock wave surfaces can be viewed close to tangency so that fringe localization is accurate. More detailed information is aval laple about

\section{Setup for Holographic Cinematography}

The setup shown in Fig. 3 is a standard offaxis-reference-beam holography setup where each hologram is exposed according to well established principles. $(8,9)$ The following specific features are of special interest, however.

Object Beam. The object beam is derived by reflecting a laser beam from the far end wall of the tunnel. The light is admitted through a window which serves as the near end wall of the tunnel. The far end wall of the tunnel is a diffuse reflector. The diffusely reflected object illumination passes through the blade passages and out the same window used to admit it. There is a small angle between the viewing direction and the blade axis.

Reference Beam. The reference beam is a diverging beam reflected to the hologram from a mirror attached to the tunnel. The apparent source of the reference beam is near the midplane of the tunnel. This is the so called lensless Fourier transform configuration for recording holograms. The main property of this configuration is that the reconstructed image will be stationary even though the hologram is moved through the reconstruction beam. Then, one can view the different frames of a holographic motion picture while moving the film continuously through the reconstruction beam.

Recording Material. The holograms are re corded on $70 \mathrm{~mm}$ film transported by a $70 \mathrm{~mm}$ motion picture camera body (Mitchell DA-70). The film is coated with Kodak SO-253 emulsion. The camera body can be operated at 10,20, or 30 frames per sec.

Laser

Description. The laser shown in Fig. 3 is a double-pulse, Q-switched, frequency-doubled Nd:YAG laser which can be operated at 20 double pulses per sec. The laser was constructed to meet Lewis's specifications for a holographic laser. An offthe-shelf laser suitable for holographic cinematography is now manufactured. The new laser will radiate at least $500 \mathrm{~mJ}$ of $532 \mathrm{~nm}$ light per double pulse, whereas the laser used for the results of this report radiates only 10 to $15 \mathrm{~mJ}$. For holography, it is convenient and desirable to have a coherence length of at least $30 \mathrm{~cm}$ (spectroscopic innewidth no larger than .03 inverse cent imeters). Intracavity etalons are used to increase the coherence length. The laser shown in Fig. 3 uses a stable resonator and radiates a TEMOO beam. (16) The individual pulses are about $10 \mathrm{~ns}$ wide, and the separation between pulses of a double pulse $c$ an be varled from 1 to 100 us in 1 us increments. In fact, vibration can degrade results if pulse separations exceed about 20 ws.

Synchronization. The holograms (frames) can be synchronized to a particular angle of attack of the blades. At $580 \mathrm{~Hz}$, a hologram rate of at least 1200 holograms per sec would be required to view the history of the shock waves in real time. At 20 holograms per second, the time variation of the shock waves is examined stroboscopically: changes from one hologram to the next represent variations not synchronized with every 29 th repetition of a particular angle of attack. Synchronization is accomplished using equipment similar to that reported elsewhere for optical measurements in rotating machines. (17) One special feature is that the angle of attack of the exposure can be programed to change automatically after a specified number of frames. The firing of the laser is synchronized with the angle of attack and a cameraready signal.

Results

In Fig. 4, the shock structure at four different angles of attack is shown. In the photographs, the shock waves look like filaments hanging from the blades. When the three-dimensional reconstructed image is viewed, the fringes corresponding to the shock structure can be seen hanging sheetlike between the endwall boundary layers. For the same angle of attack, there is no change in the shock structure from one frame to the next. However, the background changes considerably. Somet imes, the shock fringe is a dark fringe against a light background. Other $t$ imes, it is a light fringe against a dark background. Fringes corresponding to blade vibration $c$ an be seen at $t$ imes. And there are other fringes localized on tunnel turbulence. These fringes appear to be extremely well localized.

The energy of 10 to $15 \mathrm{~mJ}$ is marginal for this application, so that the new high energy laser should be especrally useful.

The hardware for double-exposure holographic c inematography is well enough developed for applications, at least at normal speed (10 to 30 holograms per second). Other systems, which are currently being studied, are discussed next.

\section{Future Directions}

The following methods of holography are being studied or considered. The objective is high-datarate three-dimensional visualization and measurement of fluid properties. 
Time-average holography will be studied using a flashlamp-pumped dye laser capable of 10 holograms per second. Exposure durations will be in the 1 to $100 \mu \mathrm{s}$ range. The intended applications are compressor, cascade, and combustion flows. Time modulation of the reference and object beams will be studied as a means for selectively enhancing flow features. The possibility of using positionally and directionally dependent modulation of the object beam for minimizing localization error will be considered. The feasibility of recording sequentially several holograms from the same laser pulse will also be examined in an attempt to lay a foundation for extremely high-data-rate or burst mode time-average holographic cinematography.

The possibility of high-speed double-exposure holographic cinematography is worth considering. A problem is that high-pulse-rate lasers such as the copper vapor laser are single-pulse lasers with pulse lengths measured in nanoseconds. The development of a convenient-to-use double-pulse system would be a major project. However, the slabgeometry solid state lasers definitely offer the possibility of 100 or more double-exposure holograms per second.

The proven Nd:YAG technology is being used for three dimensional visualization of the shock structure in Lewis's flutter cascades.

\section{Conclusions}

Both rapid-double-exposure and time-average diffuse-illumination holographic interferometry can be used for three-dimensional visualization of flow features. Although the time-average method has half the sensitivity of the double-exposure method, there is avallable the freedom of time modulating the reference and object waves. Modulation probably is not useful when the amplitude exceeds $0.6 \pi \mathrm{rad}$. during the exposure.

If the objective of holographic interferometry 15 to observe the time history of a flow feature. then its success depends entirely upon the avallability of high-pulse-rate lasers. The lasers must have the coherence and beam quality sultable for holography. An alternative is to record severa holograms sequentially during a single high-energy laser pulse.

The off-the-shelf technology for holographic cinematography is the Nd:YAG laser used at its second harmonic frequency. At around 20 holograms per second, this laser is used strobosopically to view propulsion flows. Real time holographic cinematography of propulsion flows will require in excess of 1000 holograms per second.

\section{References}

(1) Witte, A. B., and Wuerker, R. F., "Laser Holographic Interferometry Study of High Speed Flow Fields," AIAA Paper 69-347, Apr. 1969.

(2) Wuerker, R. F., Kobayashı, R. J., Heflinger, L. 0., and Ware. T. C., "Application of Holography to Flow Visualization within Rotating Compressor Blade Row." AlResearch Mfg. Co., Los Angeles, Calif., AlResearch-73-9489, Feb. 1974. (NASA CR-121264.)
(3) Decker, A. J., "Fringe Localızation Requirements for Three-Dimensional Flow Visualization of Shock Waves in

Diffuse-Illumination Double-Pulse Holographic Interferometry," NASA TP-1868, 1982.

(4) Decker, A. J., "Holographic Flow Visualization of Time-Varying Shock Waves," Applied Optics, Vol. 20, Sept. 15, 1981, pp. 3120-3127.

(5) Vest, C. M., Holographic Interferometry, Wrley, New York, 1979, pp. 107-145, pp. $177-310$.

(6) Stetson, K. A., "Effects of Beam Modulation on Fringe Loc 1 and Localization in Time-Average Hologram Interferometry," Journal of the Optical Society of America, vol. 60, Oct., 1970, pp. 1378-1388.

(7) Decker, A. J., "Holographic Cinematography of Time-Varying Reflecting and Time-Varying Phase Objects Using a Nd:YAG Laser, "Optics Letters, Vol. 7, Mar. 1982, pp. 122-123.

(8) Collier, R. J., Burckhardt, C. B., and Lin, L. H., Optical Holography, Academic Press, New York, T971.

(9) Caulfield, H. J., ed., Handbook of Optical Holography, Academic Press, New York, 1979.

(10) Howes, W. L., and Buchele, D. R., "A Theory and Method for Applying Interferometry to the Measurement of Certain Two-Dimensional Gaseous Density Frelds," NACA TN-2693, 1952.

(11) Wilson, A. D., "Characteristic Functions for Time-Average Holography," Journal of the optical Society of America, Vol. 60, Aug. 1970, pp. 1068-1071

(12) Strazısar, A. J., and Chıma, R. V. "Comparison Between Optical Measurements and a Numerical Solution of the Flow Field in a Transonic Axial-Flow Compressor Rotor," AIAA Paper 80-1078, June, 1980.

(13) Schumann, W., "Fringe Localization in Holographic Interferometry in the Case of a Transparent object with a Nonuniformly Varying Index of Refraction, "Optics Letters, Vol. 7 , Mar. 1982, pp. 119-121

(14) Weimer, D., "Pockels-Effect Cell for Gas-Flow Simulation," NASA TP-2007, 1982.

(15) Boldman, D. R., Buggele, A. E., and Decker, A. J., "Three-Dimensional Shock Structure in a Transonic Flutter Cascade," AIAA Journal. Vol. 20, Aug. 1982, pp. 1146-1148.

(16) Koechner, W., Solid-State Laser Englneering, Sprınger-Verlag, New York, 1976.

(17) Powe11, J. A., "Electronic Angular Position Encoder Apparatus, "U.S. Patent 4,171,522, oct. 16, 1979 . 


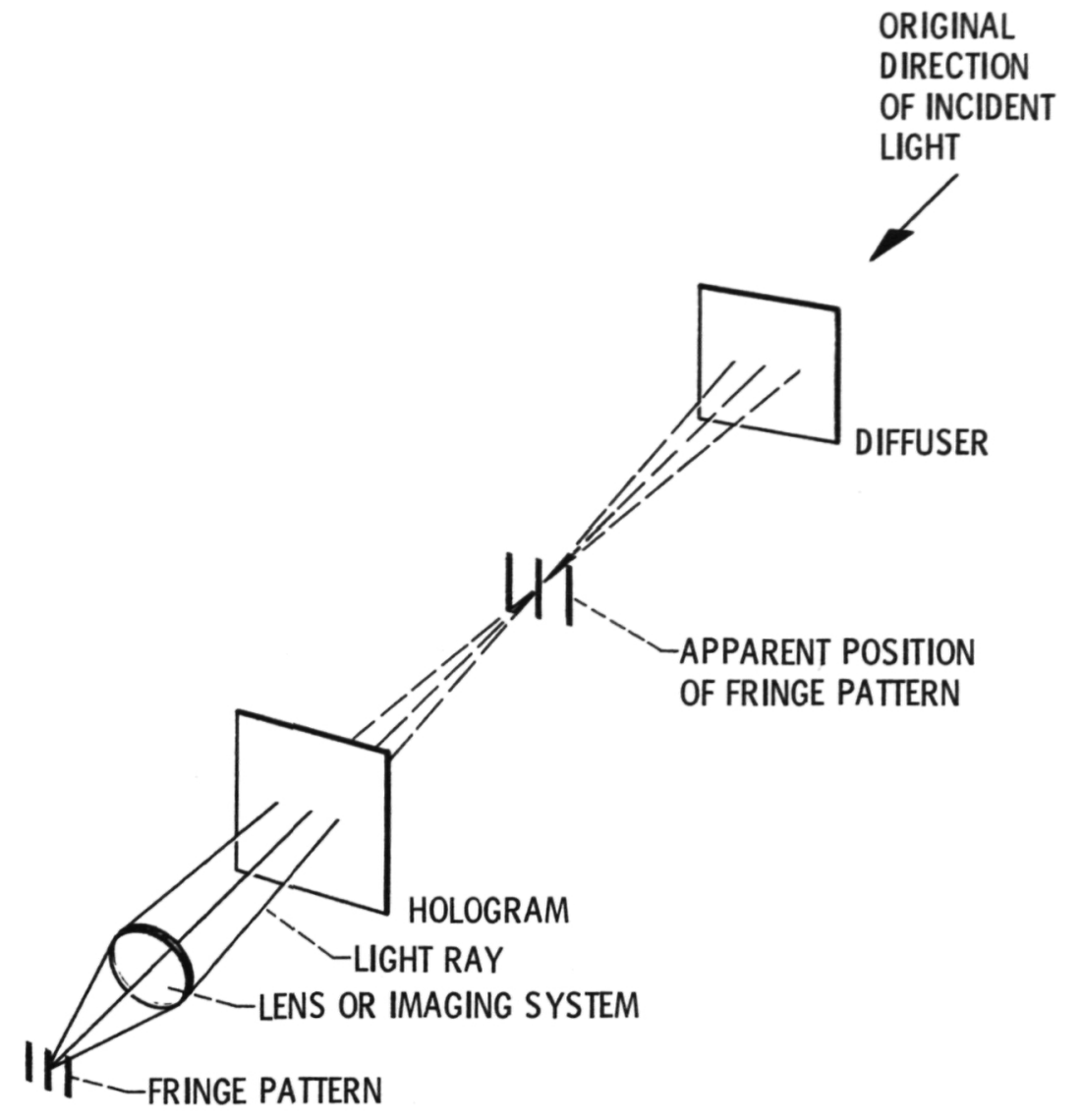

Figure 1. - Imaging a localized interference fringe pattern.

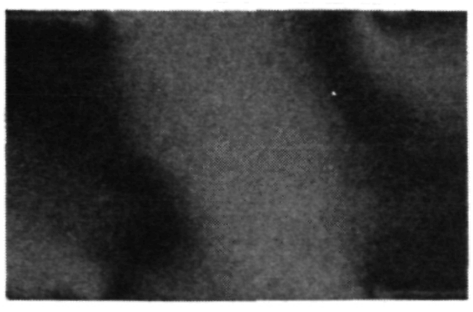

(a) Real time.

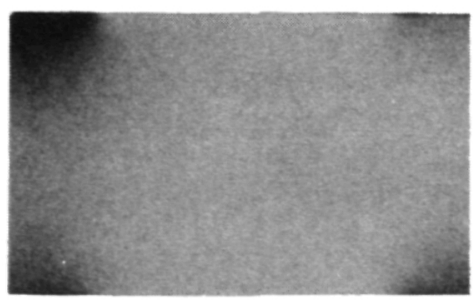

(c) Time average.

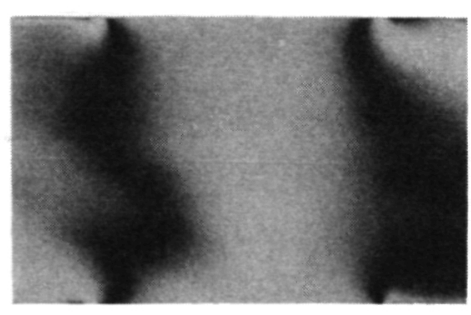

(b) Double exposure.

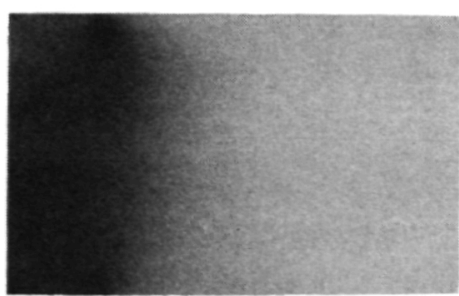

(d) Time average with frequency shifted reference beam.

Figure 2. - Flow simulator fringes. 


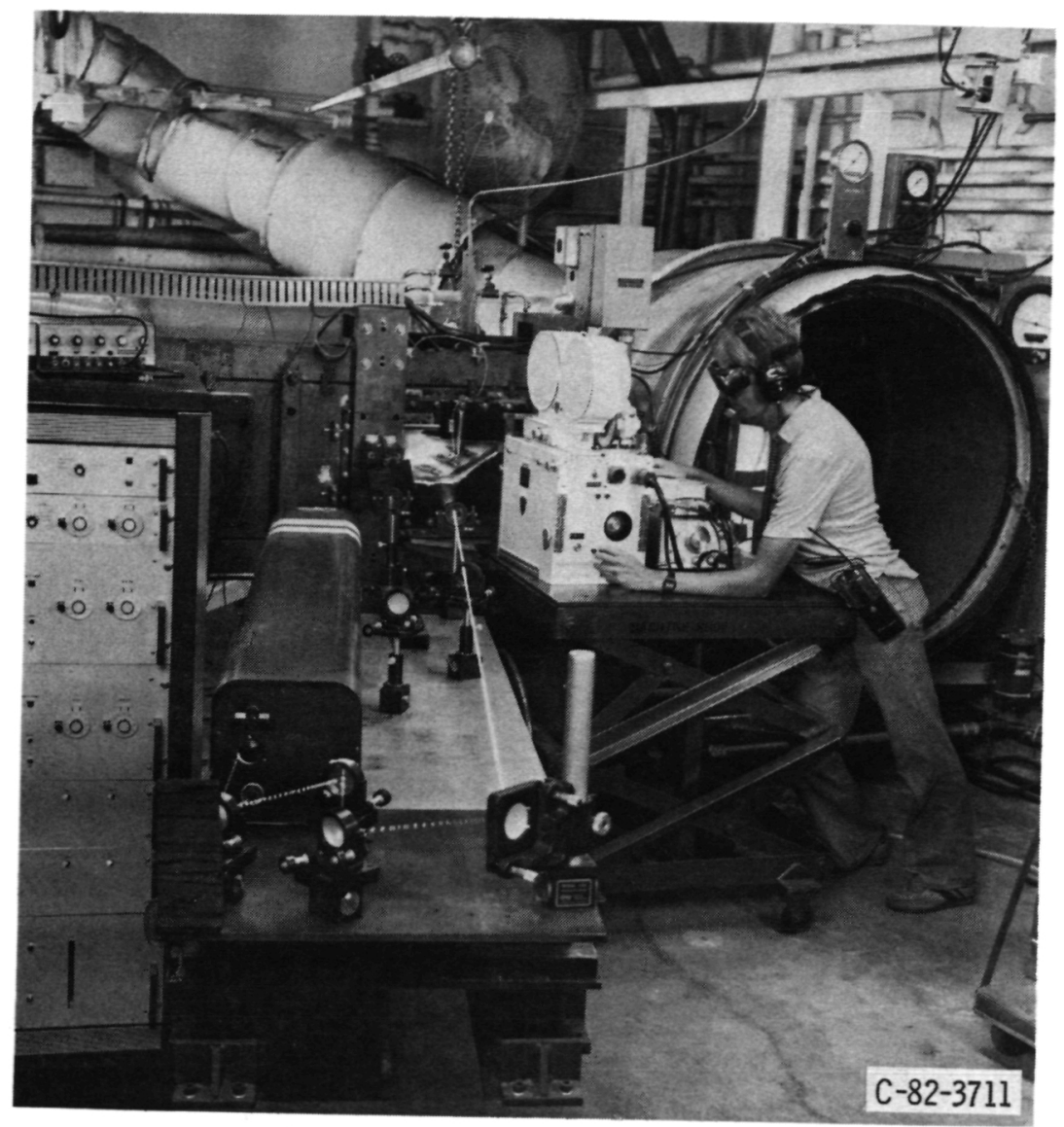

Figure 3. - Holographic cinematography in flutter cascade. 


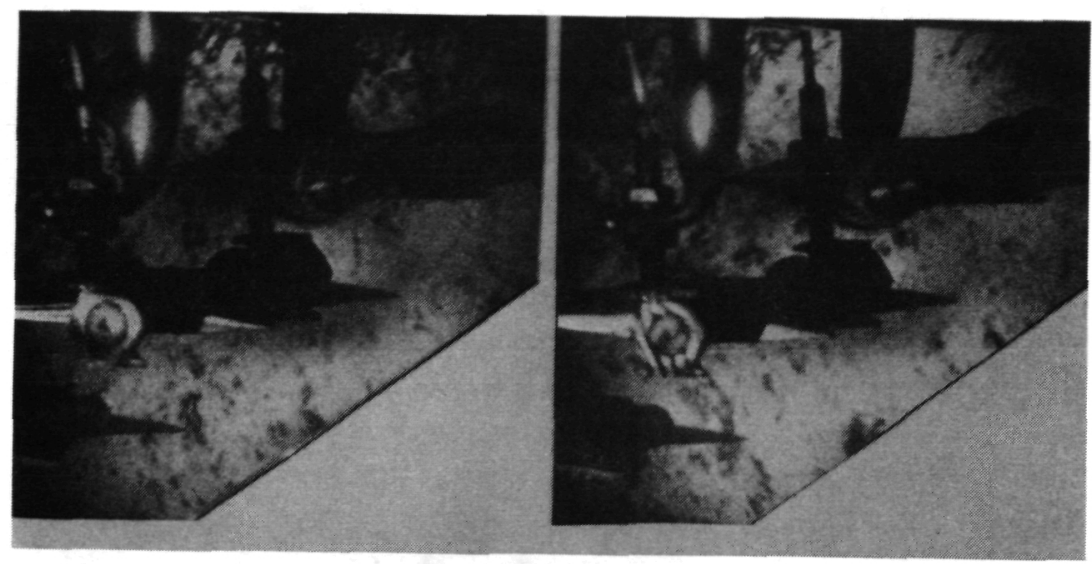

(a) Angle l.

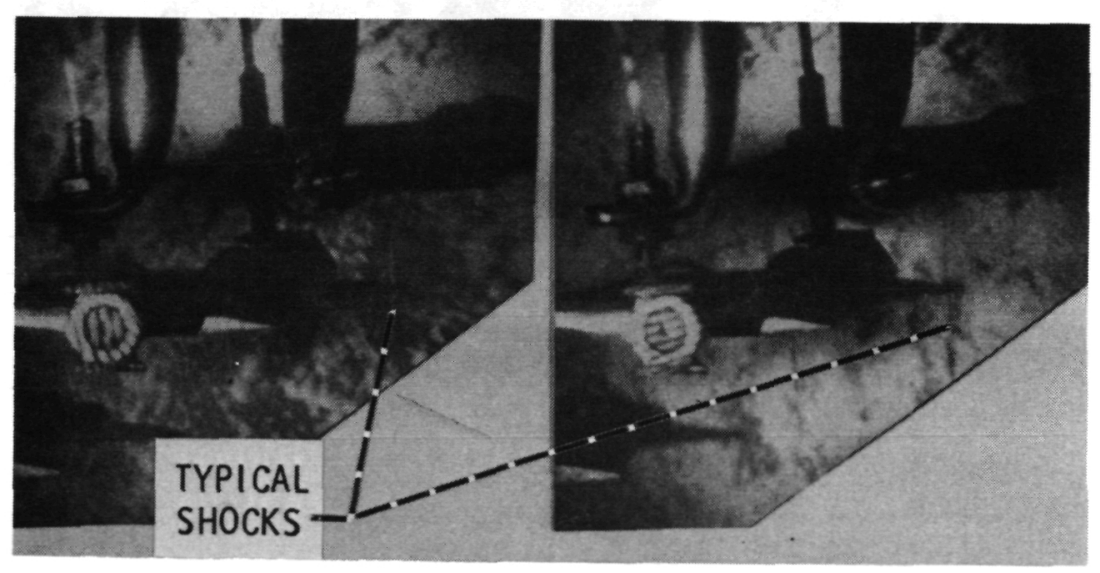

(b) Angle 2.

Figure 4. - Double exposures for several angles of attack of flutter cascade. 


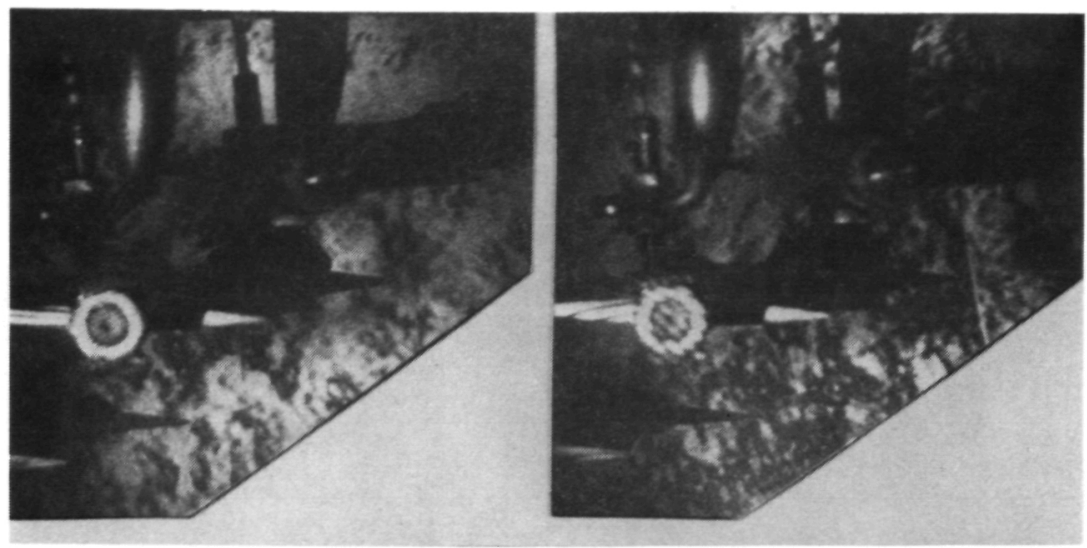

(c) Angle 3.

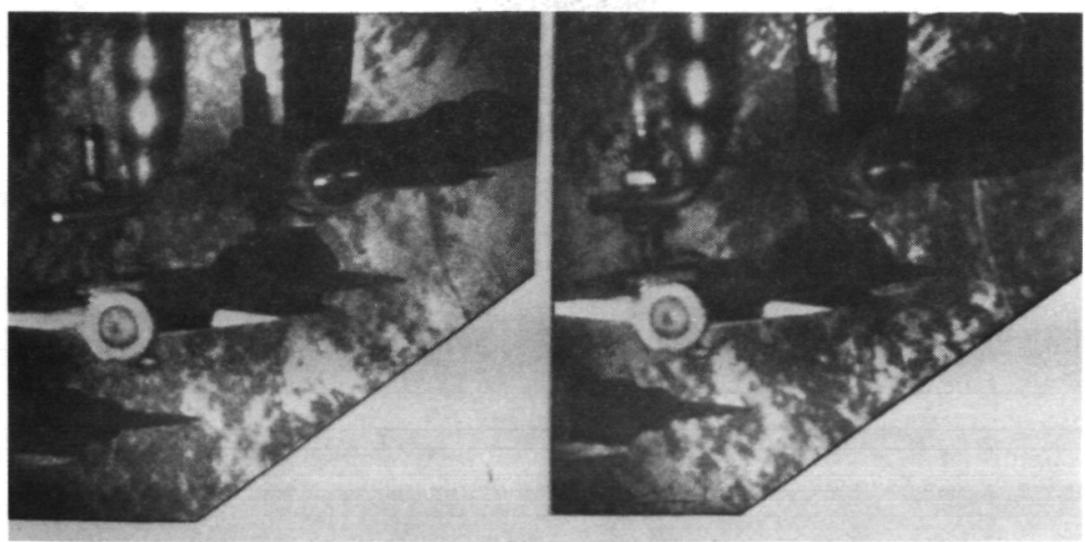

(d) Angle 4.

Figure 4. - Concluded. 


\begin{tabular}{|c|c|c|c|c|}
\hline $\begin{array}{l}1 \text { Repon No } \\
\text { NASA TM-83630 }\end{array}$ & \multicolumn{2}{|c|}{2 Government Accession No. } & \multicolumn{2}{|c|}{3 Recipient's Catalog No } \\
\hline \multicolumn{3}{|l|}{4 Title and Subtitle } & \multicolumn{2}{|l|}{5 Repon Date } \\
\hline \multicolumn{3}{|c|}{$\begin{array}{l}\text { Measurement of Fluid Properties Using Rapid-Double- } \\
\text { Exposure and Time-Average Holographic Interferometry }\end{array}$} & \multicolumn{2}{|c|}{$\begin{array}{l}6 \text { Pertorming Organization } \operatorname{Cod} \theta \\
505-31-52\end{array}$} \\
\hline \multicolumn{3}{|l|}{7 Author(s) } & \multicolumn{2}{|c|}{$\begin{array}{l}\text { 8. Pertarming Organization Repont No } \\
\text { E-2067 }\end{array}$} \\
\hline \multicolumn{3}{|l|}{ Arthur J. Decker } & \multicolumn{2}{|l|}{10 Work Unit No } \\
\hline \multirow{2}{*}{\multicolumn{3}{|c|}{$\begin{array}{l}\text { 9. Performing Organization Name and Address } \\
\text { National Aeronautics and Space Administration } \\
\text { Lewis Research Center } \\
\text { Cleveland, Ohio } 44135\end{array}$}} & \multirow{2}{*}{\multicolumn{2}{|c|}{11 Contract or Grant No }} \\
\hline & & & & \\
\hline \multirow{2}{*}{\multicolumn{3}{|c|}{$\begin{array}{l}12 \text { Sponsorno Agency Name and Address } \\
\text { National Aeronautics and Space Administration } \\
\text { Washington, D.C. } 20546\end{array}$}} & \multicolumn{2}{|c|}{$\begin{array}{l}13 \text { Type of Report and Period Covered } \\
\text { Technical Memorandum }\end{array}$} \\
\hline & & & \multicolumn{2}{|c|}{14 Sponsoring Agency Code } \\
\hline \multicolumn{5}{|c|}{$\begin{array}{l}\text { 15. Supplementay Notes } \\
\text { Prepared for the Twentieth Joint Propulsion Conference cosponsored by the AIAA, } \\
\text { SAE, and ASME, Cincinnati, Ohio, June } 11-13,1984 \text {. }\end{array}$} \\
\hline \multicolumn{5}{|c|}{$\begin{array}{l}16 \text { Abstract } \\
\text { The objective of this paper is to discuss the holographic recording of the time } \\
\text { history of a flow feature in three dimensions. The paper begins by describing } \\
\text { the use of diffuse illumination holographic interferometry for the three dimen- } \\
\text { sional visualization of flow features such as shock waves and turbulent eddies. } \\
\text { The double-exposure and time-average methods are compared using the characteris- } \\
\text { tic function and the results from a flow simulator. A time history requires a } \\
\text { large hologram recording rate. Results of holographic cinematography of the shock } \\
\text { waves in a flutter cascade are presented as an example. Future directions of } \\
\text { this effort, including the availability and development of suitable lasers, are } \\
\text { discussed. }\end{array}$} \\
\hline \multirow{2}{*}{\multicolumn{5}{|c|}{\begin{tabular}{l|l}
17 Key Words (Suggested by Author(s)) & 18 Distribution Statement \\
Holography; Holographic interferometry; & $\begin{array}{l}\text { Unclassified - unlimited } \\
\text { Flow visualization; Aircraft propulsion }\end{array}$ \\
STAR Category 35
\end{tabular}}} \\
\hline & & & & \\
\hline $\begin{array}{l}18 \text { Socurity Classit (of this report) } \\
\text { Unclassified }\end{array}$ & $\begin{array}{r}\text { Security Classif (of t } \\
\text { Uncla }\end{array}$ & ified & 21 No of pages & 22 Price $^{\circ}$ \\
\hline
\end{tabular}


National Aeronautics and

Space Administration

Washington, D.C.

20546

Offrcuat Businam

Penaliv for Privete Un. 2500
Eccial rountu clars mall

cook

1

Peacesen and foes Pold Mationst Aeroneuties und Epees Administrotion MAsa-45i 